\title{
The Exact Endoscopic Effect on the Peristaltic Flow of a Nanofluid
}

\author{
S. M. Khaled, ${ }^{1,2}$ Abdelhalim Ebaid, ${ }^{3}$ and Fahd Al Mutairi ${ }^{4}$ \\ ${ }^{1}$ Department of Mathematics, Faculty of Sciences, Helwan University, Cairo, Egypt \\ ${ }^{2}$ Department of Studies and Basic Sciences, Faculty of Community, University of Tabuk, Saudi Arabia \\ ${ }^{3}$ Department of Mathematics, Faculty of Science, University of Tabuk, Tabuk 71491, Saudi Arabia \\ ${ }^{4}$ Department of Chemical Engineering, Faculty of Engineering, University of Tabuk, Saudi Arabia
}

Correspondence should be addressed to Abdelhalim Ebaid; aebaid@ut.edu.sa

Received 17 July 2014; Revised 16 October 2014; Accepted 26 October 2014; Published 18 November 2014

Academic Editor: Nicolae Herisanu

Copyright (C) 2014 S. M. Khaled et al. This is an open access article distributed under the Creative Commons Attribution License, which permits unrestricted use, distribution, and reproduction in any medium, provided the original work is properly cited.

\begin{abstract}
The problem of the peristaltic flow of a nanofluid under the effect of an endoscope is reinvestigated. The mathematical model is governed by a system of linear and nonlinear partial differential equations with prescribed boundary conditions. Really, the exact solution for any physical problem, if available, is of great importance which inevitably leads to a better understanding of the behaviour of the involved physical phenomena. An attempt for doing so has been done in the present paper, where the temperature equation is solved exactly by the help of Laplace transform and, accordingly, the exact expressions for the nanoparticle concentration, the axial velocity, the pressure gradient, and the pressure rise are established. Furthermore, it is showed in this paper that the physical interpretations of some involved phenomena are found totally different than those previously obtained by the approximate solutions using the homotopy perturbation method. In addition, several comparisons between the current results and the approximate ones have been displayed. Finally, the effect of various parameters on the temperature distribution, the nanoparticle concentration, the pressure gradient, and the pressure rise has been also discussed through graphs.
\end{abstract}

\section{Introduction}

The subject of peristaltic flow of classical Newtonian and non-Newtonian fluids has a long history [1-7]. It was and is still of interest to many researchers because of its medical and engineering applications. For example, Mekheimer and Abd elmaboud [8] showed that the peristaltic flow of blood with bioheat transfer is of great importance in destroying undesirable tissues, such as cancer. It was also clarified in [9] that, in the case of hyperthermia, the tissue can be destroyed when heated to $42-45^{\circ} \mathrm{C}$. In addition, peristaltic flow occurs widely in the functioning of the ureter, chyme movement in the intestine, movement of eggs in the fallopian tube, the transport of the spermatozoa in cervical canal, transport of bile in the bile duct, transport of cilia, circulation of blood in small blood vessels, and the transport of intrauterine fluid within the cavity of the uterus.

Nowadays, nanotechnology is widely used in industry since materials with sizes of nanometers possess unique physical and chemical properties. Normally, if the particle sizes are in the $1-100 \mathrm{~nm}$ ranges, they are generally called nanoparticles, $1 \mathrm{~nm}$ (nano-meter) $=10^{-9}$ meters. Nanoscale particle added fluids are called nanofluid. The term nanofluid was first used by Choi [10] to describe a fluid in which nanometer-sized particles are suspended in conventional heat transfer basic fluids. Fluids such as oil, water, and ethylene glycol mixture are poor heat transfer fluids, since the thermal conductivity of these fluids plays an important role in the heat transfer coefficient between the heat transfer medium and the heat transfer surface. Numerous methods have been followed to improve the thermal conductivity of these fluids by suspending nano/micro or larger-sized particle materials in liquids. An innovative technique to improve heat transfer is by using nanoscale particles in the base fluid [10]. Choi et al. [11] showed that the addition of a small amount (less than $1 \%$ by volume) of nanoparticles to conventional heat transfer liquids increased the thermal conductivity of the fluid up to approximately two times. This 


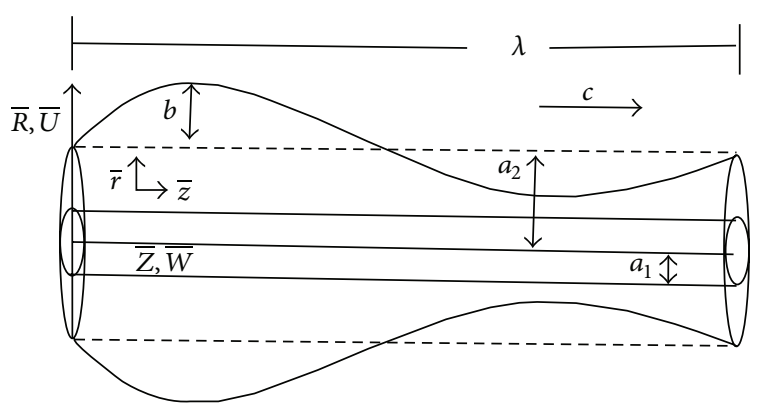

FIGURE 1: Geometry of the problem.

phenomenon suggests the possibility of using nanofluids in advanced nuclear systems (see [12]). Despite the fact that a huge number of studies are now available for the peristaltic flow of classical fluids, the first study on the peristaltic flow of a nanofluid has been introduced very recently and discussed by Akbar and Nadeem [13]. Till this moment, a few papers were presented in this area of research [13-17]. The main observation on these studies is that the authors usually resort to the approximate solutions to interpret the behaviour of the physical quantities. In fact, such approximate solutions, sometimes, do not provide us with the correct physical discussion if compared with the exact solutions [18-20]. Many useful results for nanofluids flow can be found in [2127]. In [13], Akbar and Nadeem have analyzed the peristaltic flow and heat transfer of a nanofluid in an endoscope. They have used the homotopy perturbation method to obtain the analytical approximate solutions for the temperature distribution and the nanoparticle concentration. However, their approximate solutions do not give the correct behaviour of the physical quantities as will be clarified later. So, this problem will be reinvestigated in the current paper and our objectives can be summarized as follows.

(i) The first task is to obtain the exact solutions for the system of partial differential equations governing the flow.

(ii) The obtained exact solutions are used to introduce various plots for the exact temperature and the exact nanoparticle concentration.

(iii) The final major task is to explain the big difference in the results and plots obtained in the current study using the exact solutions when compared to those obtained through the approximate solutions in [13]. This may refer to the fact that the approximate solutions do not always lead to the correct interpretations of the involved physical phenomena.

\section{The Physical Problem}

In [13], Akbar and Nadeem considered the peristaltic flow of an incompressible nanofluid in an endoscope. The flow is generated by sinusoidal wave trains propagating with constant speed $c$ along the walls of the tube. Heat transfer along with nanoparticle phenomena has been taken into account. The inner tube is rigid and is maintained at temperature $T_{0}$ while the outer tube has a sinusoidal wave traveling down its walls and is maintained at temperature $T_{1}$. The geometry of the wall surfaces, see Figure 1, is defined as

$$
\begin{aligned}
& \overline{R_{1}}=a_{1}, \\
& \overline{R_{2}}=a_{1}+b \sin \left[\frac{2 \pi}{\lambda}(\bar{Z}-c \bar{t})\right],
\end{aligned}
$$

where $a_{1}$ is the radius of the inner tube, $a_{2}$ is the radius of the outer tube at inlet, $b$ is the wave amplitude, $\lambda$ is the wavelength, and $t$ is the time. Introducing a wave frame $(\bar{r}, \bar{z})$ moving with velocity $c$ away from the fixed frame $(\bar{R}, \bar{Z})$ by the transformations

$$
\bar{z}=\bar{Z}-c \bar{t}, \quad \bar{r}=\bar{R}, \quad \bar{w}=\bar{W}-c, \quad \bar{u}=\bar{U},
$$

in which $\bar{U}, \bar{W}$ and $\bar{u}, \bar{w}$ are the velocity components in the radial and axial directions in the fixed and moving coordinates, respectively. In their analysis, it was found that under the assumptions of long wavelength and low Reynolds number approximation the flow is governed by the following system of partial differential equations in nondimensional form [13]:

$$
\begin{gathered}
\frac{\partial u}{\partial r}+\frac{u}{r}+\frac{\partial w}{\partial z}=0 \\
\frac{\partial P}{\partial r}=\frac{1}{r} \frac{\partial}{\partial r}\left(r \frac{\partial w}{\partial r}\right)+G_{r} \theta+B_{r} \sigma \\
\frac{1}{r} \frac{\partial}{\partial r}\left(r \frac{\partial \theta}{\partial r}\right)+N_{b} \frac{\partial \sigma}{\partial r} \frac{\partial \theta}{\partial r}+N_{t}\left(\frac{\partial \theta}{\partial r}\right)^{2}=0 \\
\frac{1}{r} \frac{\partial}{\partial r}\left(r \frac{\partial \sigma}{\partial r}\right)+\frac{N_{t}}{N_{b}}\left[\frac{1}{r} \frac{\partial}{\partial r}\left(r \frac{\partial \theta}{\partial r}\right)\right]=0,
\end{gathered}
$$

where $N_{b}, N_{t}, G_{r}$, and $B_{r}$ are, respectively, the Brownian motion parameter, the thermophoresis parameter, the local temperature Grashof number, and the nanoparticle Grashof number. The flow is subject to the boundary conditions:

$$
\begin{array}{r}
\begin{array}{r}
w=-1, \quad \\
\text { at } r=r_{1}=\epsilon,
\end{array} \\
w=-1, \quad \theta=0, \quad \sigma=0, \\
\text { at } r=r_{2}=1+\phi \sin (2 \pi z) .
\end{array}
$$

In nondimensional form [13], the transformation $\bar{w}=\bar{W}-c$ becomes $w=W-1$. Therefore, the no-slip condition at the wall $W=0$ leads to the boundary condition $w=-1$ in the moving frame. The system ((3)-(8)) was solved approximately in [13] by using the homotopy perturbation method. The approximate series solution obtained in [13] was used to discuss various physical phenomena such as temperature profile, nanoparticle concentration, pressure gradient, and the streamlines. However, the exact solutions of the above system can be obtained and this is the subject of the next section. 


\section{The Exact Solutions}

At first, (5) reveals that the axial velocity depends on getting the temperature and the concentration. So, it may be useful to first focus on searching for the exact solutions to (6) and (7). It is observed from (7) that it can be easily integrated twice with respect to $r$ to give the following exact relation between the temperature and the nanoparticle concentration:

$$
\sigma(r, z)=h_{1}(z) \ln r-\left(\frac{N_{t}}{N_{b}}\right) \theta+h_{2}(z),
$$

where $h_{1}(z)$ and $h_{2}(z)$ are two unknown functions. On applying the boundary conditions in (8) to (9), we obtain

$$
h_{1}(z)=\frac{1+N_{t} / N_{b}}{\ln \left(N_{t} / N_{b}\right)}, \quad h_{2}(z)=-\left(\frac{1+N_{t} / N_{b}}{\ln \left(N_{t} / N_{b}\right)}\right) \ln \left(r_{2}\right) .
$$

Substituting (9) into (6) yields a partial differential equation in only $\theta$ :

$$
\frac{1}{r} \frac{\partial}{\partial r}\left(r \frac{\partial \theta}{\partial r}\right)+N_{b} h_{1}(z) \frac{\partial \theta}{\partial r}=0,
$$

which can be integrated with respect to $r$ once again to give

$$
r \frac{\partial \theta}{\partial r}+N_{b} h_{1}(z) \theta=h_{3}(z),
$$

where $h_{3}(z)$ is also an unknown function and is to be determined later. In order to solve (12), a new independent variable $x$ is suggested, where $x=\ln (r)$ or $r=e^{x}$. Accordingly, (12) becomes

$$
\frac{\partial \theta}{\partial x}+N_{b} h_{1}(z) \theta(x, z)=h_{3}(z) .
$$

Equation (13) can be now solved exactly by the help of Laplace transform as follows. Taking Laplace transform to both sides of the last partial differential equation, we get

$$
\widetilde{\theta}(x, z)=\frac{\theta(0, z)}{s+N_{b} h_{1}(z)}+\frac{h_{3}(z)}{s\left(s+N_{b} h_{1}(z)\right)},
$$

where $\theta(0, z)$ is an unknown function. Applying the inverse Laplace transform to (14) yields

$$
\theta(x, z)=\theta(0, z) e^{-N_{b} h_{1}(z) x}+\frac{h_{3}(z)}{N_{b} h_{1}(z)}\left[1-e^{-N_{b} h_{1}(z) x}\right] .
$$

In terms of the original independent variable $r$ we obtain

$$
\theta(r, z)=\theta(1, z) r^{-N_{b} h_{1}(z)}+\frac{h_{3}(z)}{N_{b} h_{1}(z)}\left[1-r^{-N_{b} h_{1}(z)}\right] .
$$

The unknown functions $\theta(1, z)$ and $h_{3}(z)$ can be now determined by applying the boundary conditions to (16). Therefore

$$
h_{3}(z)=\frac{N_{b} h_{1}(z)}{1-\left(r_{2} / r_{1}\right)^{N_{b} h_{1}(z)}}, \quad \theta(1, z)=\frac{1-\left(r_{2}\right)^{N_{b} h_{1}(z)}}{1-\left(r_{2} / r_{1}\right)^{N_{b} h_{1}(z)}},
$$

and, hence, the exact analytical form for the temperature distribution is given by

$$
\theta(r, z)=\frac{1-\left(r / r_{2}\right)^{N_{b} h_{1}(z)}}{1-\left(r_{1} / r_{2}\right)^{N_{b} h_{1}(z)}} .
$$

Here, it should be noted that the exact solution obtained above and given by (18) can be easily verified by the direct substitution. Moreover, the exact analytical expression for the nanoparticle concentration is obtained by substituting (18) into (9) as

$$
\sigma(r, z)=h_{1}(z) \ln \left(\frac{r}{r_{2}}\right)\left(\frac{N_{t}}{N_{b}}\right)\left(\frac{1-\left(r / r_{2}\right)^{N_{b} h_{1}(z)}}{1-\left(r_{1} / r_{2}\right)^{N_{b} h_{1}(z)}}\right),
$$

where $h_{1}(z)$ is already defined in (10). Our task now is to construct the exact solution for the axial velocity $w(r, z)$. This task is achieved as follows. In view of (4), the pressure $P$ becomes a function in only $z$, that is, $P=P(z)$, and accordingly (5) can be written as

$$
\frac{1}{r} \frac{\partial}{\partial r}\left(r \frac{\partial w}{\partial r}\right)=\frac{d P}{d z}-G_{r} \theta-B_{r} \sigma,
$$

which is also integrated twice with respect to $r$ to give

$$
\begin{aligned}
w(r, z)= & \frac{1}{4} \frac{d P}{d z} r^{2}-G_{r} \Pi_{1}(r, z)-B_{r} \Pi_{2}(r, z) \\
& +h_{4}(z) \ln (r)+h_{5}(z) .
\end{aligned}
$$

Here $h_{4}(z)$ and $h_{5}(z)$ are two further unknown functions and $\Pi_{1}(r, z)$ and $\Pi_{2}(r, z)$ are defined by the following indefinite integrals:

$$
\begin{aligned}
& \Pi_{1}(r, z)=\int\left[\frac{1}{r} \int r \theta(r, z) d r\right] d r, \\
& \Pi_{2}(r, z)=\int\left[\frac{1}{r} \int r \sigma(r, z) d r\right] d r .
\end{aligned}
$$

On performing the above integrations, we obtain

$$
\begin{aligned}
& \Pi_{1}(r, z)=\frac{r^{2}}{1-\left(r_{1} / r_{2}\right)^{-N_{b} h_{1}(z)}}\left[\frac{1}{4}-\frac{\left(r / r_{2}\right)^{-N_{b} h_{1}(z)}}{\left(2-N_{b} h_{1}(z)\right)^{2}}\right], \\
& \Pi_{2}(r, z)=\frac{1}{4} h_{1}(z) r^{2}\left[\ln \left(\frac{r}{r_{2}}\right)-1\right]-\left(\frac{N_{t}}{N_{b}}\right) \Pi_{1}(r, z) .
\end{aligned}
$$

In view of (21) and (24), the exact expression for $w(r, z)$ can be written as

$$
\begin{aligned}
w(r, z)= & \frac{r^{2}}{4}\left[\Lambda(z)-B_{r} h_{1}(z) \ln \left(\frac{r}{r_{2}}\right)\right]-\Omega \Pi_{1}(r, z) \\
& +h_{4}(z) \ln r+h_{5}(z),
\end{aligned}
$$

where $\Lambda(z)$ and $\Omega$ (a constant) are given as

$$
\Lambda(z)=\frac{d P}{d z}+B_{r} h_{1}(z), \quad \Omega=G_{r}-\frac{B_{r} N_{t}}{N_{b}} .
$$


Applying the boundary conditions (8) to (25), it then follows that

$$
\begin{aligned}
& \frac{r_{1}^{2}}{4}\left[\Lambda(z)-B_{r} h_{1}(z) \ln \left(\frac{r_{1}}{r_{2}}\right)\right]-\Omega \Pi_{1}\left(r_{1}, z\right) \\
& \quad+h_{4}(z) \ln r_{1}+h_{5}(z)=-1, \\
& \frac{r_{2}^{2}}{4} \Lambda(z)-\Omega \Pi_{1}\left(r_{2}, z\right)+h_{4}(z) \ln r_{2}+h_{5}(z)=-1 .
\end{aligned}
$$

Solving this system for $h_{4}(z)$ and $h_{5}(z)$, we get

$$
\begin{aligned}
& h_{4}(z) \\
& =\frac{B_{r}}{4} h_{1}(z) r_{1}^{2} \\
& +\frac{\left[(\Lambda(z) / 4)\left(r_{2}^{2}-r_{1}^{2}\right)+\Omega\left(\Pi_{1}\left(r_{1}, z\right)-\Pi_{1}\left(r_{2}, z\right)\right)\right]}{\ln \left(r_{1} / r_{2}\right)} \\
& h_{5}(z)=-1-\frac{1}{4} \Lambda(z) r_{2}^{2}+\Omega \Pi_{1}\left(r_{1}, z\right)-h_{4}(z) \ln \left(r_{2}\right) \text {. }
\end{aligned}
$$

Therefore, the axial velocity $w(r, z)$ is given by

$w(r, z)$

$$
\begin{aligned}
= & -1+\frac{1}{4} \Lambda(z)\left(r^{2}-r_{2}^{2}\right)+\left(h_{4}(z)-\frac{1}{4} B_{r} h_{1}(z) r^{2}\right) \ln \left(\frac{r}{r_{2}}\right) \\
& +\Omega\left(\Pi_{1}\left(r_{2}, z\right)-\Pi_{1}\left(r_{1}, z\right)\right) .
\end{aligned}
$$

By substituting $h_{4}(z)$ given in (28) into (30), we obtain the following exact expression for the axial velocity:

$$
\begin{aligned}
& w(r, z) \\
& =-1-\frac{B_{r}}{4} h_{1}(z) \Lambda(z)\left(r^{2}-r_{1}^{2}\right) \ln \left(\frac{r}{r_{2}}\right) \\
& +\frac{\Lambda(z)}{4}\left[r^{2}-r_{2}^{2}+\left(\frac{r_{2}^{2}-r_{1}^{2}}{\ln \left(r_{1} / r_{2}\right)}\right) \ln \left(\frac{r}{r_{2}}\right)\right] \\
& +\Omega\left[\Pi_{1}\left(r_{2}, z\right)-\Pi_{1}(r, z)\right. \\
& \left.+\left(\frac{\Pi_{1}\left(r_{2}, z\right)-\Pi_{1}\left(r_{2}, z\right)}{\ln \left(r_{1} / r_{2}\right)}\right) \ln \left(\frac{r}{r_{2}}\right)\right] .
\end{aligned}
$$

According to [25], the pressure gradient is given by

$$
\frac{d P}{d z}=\frac{F-\Gamma_{1}(z)}{\Gamma_{2}(z)}-B_{r} h_{1}(z),
$$

where the flow rate in dimensionless form is defined as

$$
F=2 Q-\frac{\phi}{2}-1
$$

Besides, $\Gamma_{1}(z)$ and $\Gamma_{2}(z)$ are given as follows:

$$
\begin{aligned}
& \Gamma_{1}(z) \\
& =\int_{r_{1}}^{r_{2}}\left[-2 r-\frac{1}{2} B_{r} h_{1}(z)\left(r^{3}-r_{1}^{2} r\right) \ln \left(\frac{r}{r_{2}}\right)\right] d r+2 \Omega \\
& \times \int_{r_{1}}^{r_{2}}\left[\frac{\Pi_{1}\left(r_{1}, z\right)}{\ln \left(r_{1} / r_{2}\right)} \times r \ln \left(\frac{r}{r_{2}}\right)-\frac{\Pi_{1}\left(r_{2}, z\right)}{\ln \left(r_{1} / r_{2}\right)}\right. \\
& \left.\quad \times r \ln \left(\frac{r}{r_{1}}\right)-r \Pi_{1}(r, z)\right] d r, \\
& \Gamma_{2}(z)=\frac{1}{2} \int_{r_{1}}^{r_{2}}\left[r^{3}-r_{2}^{2} r+\left(\frac{r_{2}^{2}-r_{1}^{2}}{\ln \left(r_{1} / r_{2}\right)}\right) \times r \ln \left(\frac{r}{r_{2}}\right)\right] d r .
\end{aligned}
$$

The pressure rise in nondimensional form is expressed as

$$
\Delta P=\int_{0}^{1} \frac{d P}{d z} d z=\int_{0}^{1}\left[\frac{2 Q-\phi / 2-1-\Gamma_{1}(z)}{\Gamma_{2}(z)}-B_{r} h_{1}(z)\right] d z .
$$

In [13], the authors obtained the approximate solutions for the temperature distribution and the nanoparticle concentration as

$$
\begin{aligned}
& \theta_{\mathrm{HPM}}(r, z) \\
& \begin{aligned}
=\frac{1}{A_{1}}[ & A_{2}\left(\log r-\log r_{1}\right)\left(2 \log r-A_{4}\right) \\
& -6\left(N_{b}+N_{t}\right)\left(\log r-\log r_{1}\right)\left(\log r-\log r_{2}\right) \\
& \left.+12 A_{4}^{2}\left(\log r-\log r_{2}\right)\right],
\end{aligned}
\end{aligned}
$$

$$
\begin{aligned}
\sigma_{\mathrm{HPM}}(r, z) & \\
=\frac{1}{A_{4} A_{5}}[ & A_{5}\left(\log r-\log r_{2}\right)+A_{3} A_{4}\left(\log r-\log r_{1}\right) \\
& \left.\times\left(\log r-\log r_{2}\right)\left(2 \log r-A_{4}\right)\right],
\end{aligned}
$$

where

$$
\begin{aligned}
& A_{1}=12\left(\log r_{1}-\log r_{2}\right)^{3}, \\
& A_{2}=-\left(N_{b}+N_{t}\right)\left(N_{b}+2 N_{t}\right), \\
& A_{3}=N_{t}\left(N_{b}+N_{t}\right)\left(N_{b}+2 N_{t}\right), \\
& A_{4}=\log r_{1}-\log r_{2}, \\
& A_{5}=12 N_{b}\left(\log r_{1}-\log r_{2}\right)^{3} .
\end{aligned}
$$

\section{Numerical Results}

In the previous section, the exact solutions for the temperature, nanoparticle concentration, the axial velocity, the pressure gradient, and the pressure rise have been obtained. 


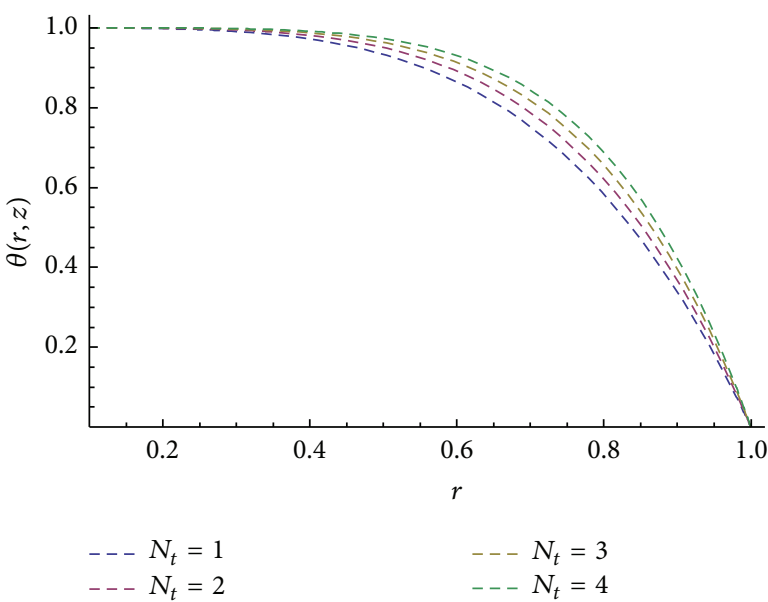

(a)

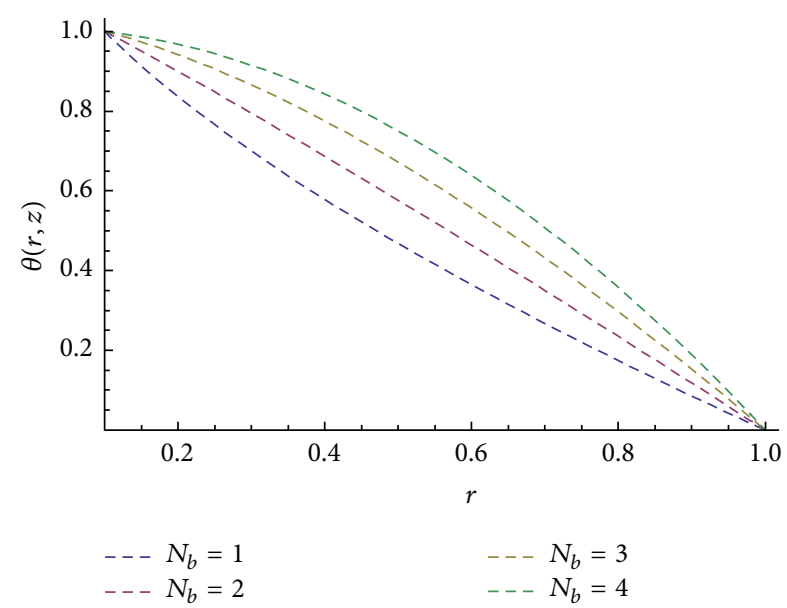

(b)

Figure 2: (a) Temperature profile for different values of $N_{t}$ at $N_{b}=8, r_{1}=0.1, \phi=0.2$, and $z=0.5$. (b) Temperature profile for different values of $N_{b}$ at $N_{t}=0.5, r_{1}=0.1, \phi=0.2$, and $z=0.5$.

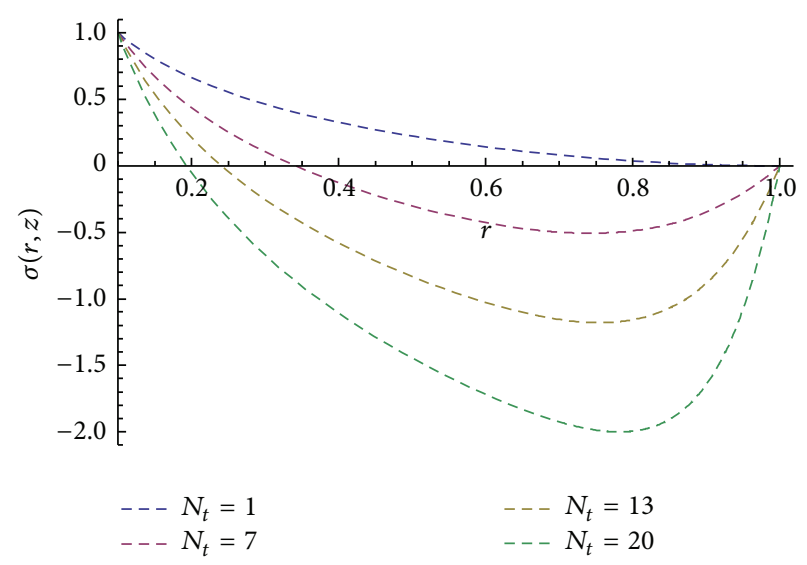

(a)

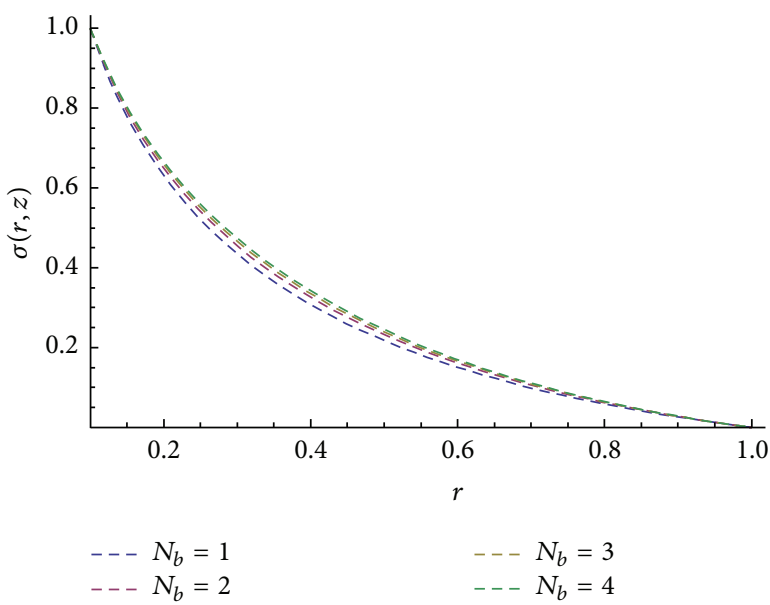

(b)

Figure 3: (a) Concentration profile for different values of $N_{t}$ at $N_{b}=8, r_{1}=0.1, \phi=0.2$, and $z=0.5$. (b) Concentration profile for different values of $N_{b}$ at $N_{t}=0.5, r_{1}=0.1, \phi=0.2$, and $z=0.5$.

Undoubtedly, such exact solutions are of great importance and certainly would lead to a better understanding of the physical aspects of the model. In the present section, the obtained exact solutions are used to explore the actual effects of various parameters on the temperature distribution, the nanoparticle concentration, the pressure gradient, and the pressure rise.

Figures 2(a) and 2(b) represent the temperature profiles at different values of the physical parameters. Figure 2(a) indicates that the temperature profile decreases in the whole region $0.1 \leq r \leq 1$ at different values of the thermophoresis parameter $N_{t}$. Unfortunately, the results presented in Figure 2(a) contradict those obtained by Akbar and Nadeem [13] (Figure 5(a)) at the same values of the physical parameters. In addition, in [13], the authors used the approximate solutions derived from the homotopy perturbation method to show in Figure 5(a) that the temperature profile increases in the region $0.1 \leq r \leq 0.41$ and decreases in the region $0.41 \leq r \leq 1$. This may refer to the fact that their approximate solutions were not really effective. In Figure 2(b) an additional figure for the temperature profile is depicted at different values of the Brownian motion parameter $N_{b}$, where the temperature profile also decreases in the whole region $0.1 \leq r \leq 1$. Comparing the results presented in the current study in Figure 2(b) with those obtained in Figure 5(b) in [13] at the same parameters values one can easily observe the big difference between the temperature curves. This difference in results can be also observed through comparing Figures 3(a) and 3(b) with Figures 6(a) and 6(b) in [13] for the nanoparticle concentration profile.

Due to this remarkable difference in the behaviour of the mentioned physical quantities, it may be concluded that the approximate solutions obtained in [13] were not effective enough to give the correct physical interpretation. Therefore, 


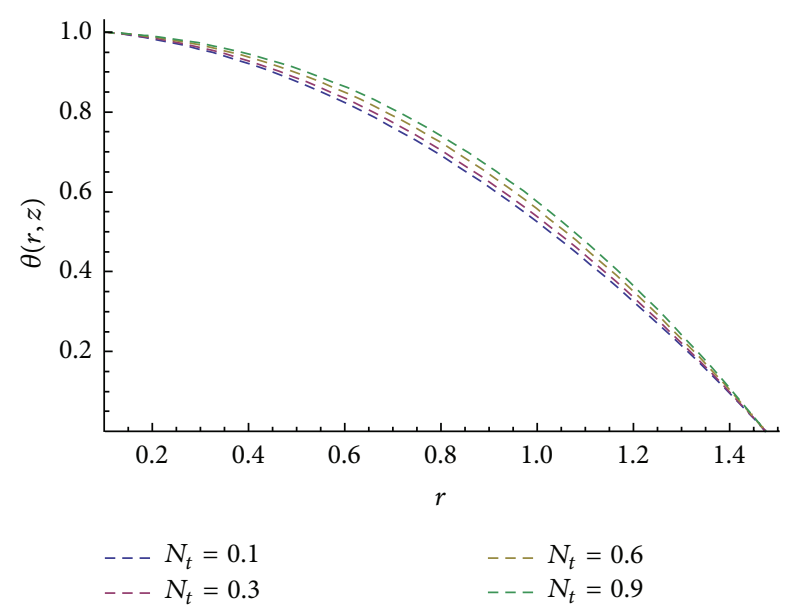

FIgURE 4: Temperature profile for different values of $N_{t}$ at $N_{b}=5$, $r_{1}=0.1, \phi=0.5$, and $z=0.3$.

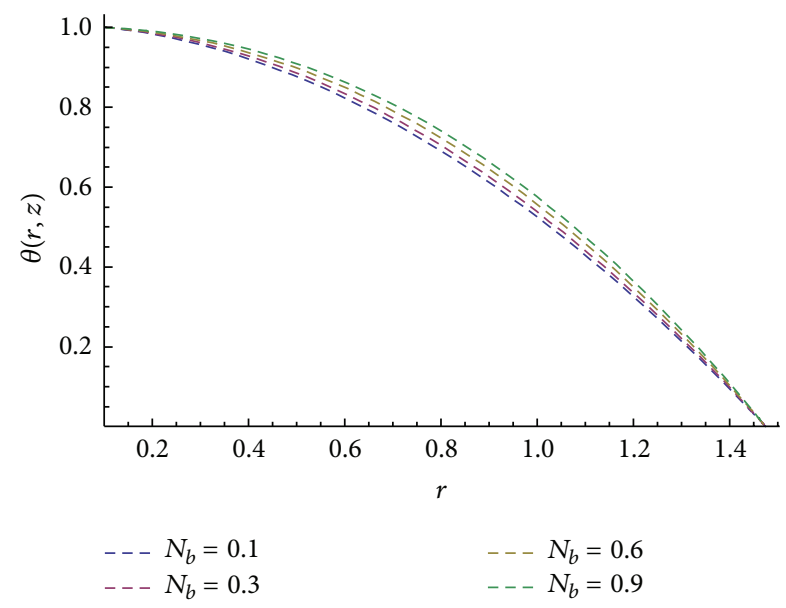

Figure 5: Temperature profile for different values of $N_{b}$ at $N_{t}=5$, $r_{1}=0.1, \phi=0.5$, and $z=0.3$.

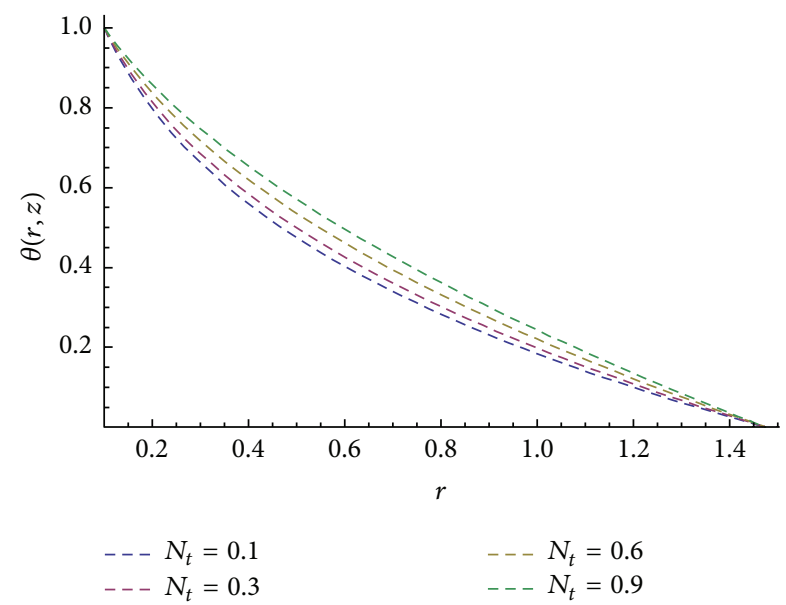

Figure 6: Temperature profile for different values of $N_{t}$ at $N_{b}=0.5$, $r_{1}=0.1, \phi=0.5$, and $z=0.3$.

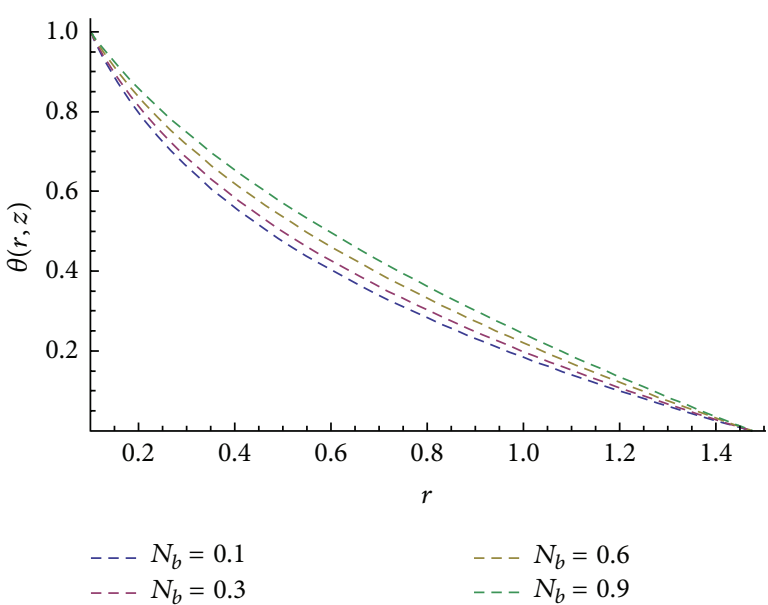

FIgURE 7: Temperature profile for different values of $N_{b}$ at $N_{t}=0.5$, $r_{1}=0.1, \phi=0.5$, and $z=0.3$.

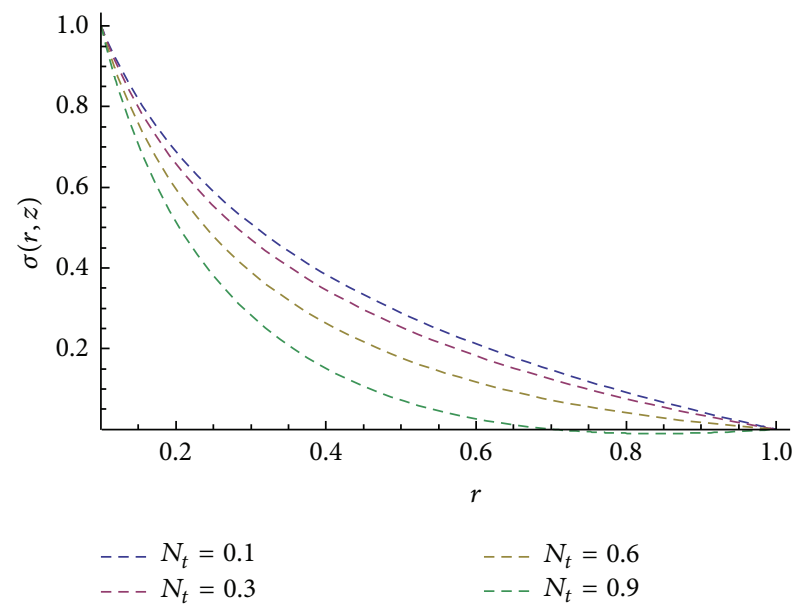

Figure 8: Concentration profile for different values of $N_{t}$ at $N_{b}=$ $0.7, r_{1}=0.1, \phi=0.2$, and $z=0.5$.

the obtained exact solutions should be invested here. To do so, several plots are presented in Figures 4-9 for the variation of $\theta$ and $\sigma$ versus $r$ at additional values for the parameters $N_{t}$ and $N_{b}$. Here, it may be important to mention that the chosen values for $N_{t}$ and $N_{b}$ as observed in [13] were always exceeding one. However, Rana and Bhargava [28] and Makinde and Aziz [29] as well as Aly et al. [30] practically studied $N_{b}$ and $N_{t}$ in the range of 0.1-0.5. Hence, the variation of these nondimensional parameters is considered here to vary in wider ranges than those mentioned above. Figures 4-7 indicate that the behaviour of the temperature mainly depends on whether $N_{t}$ or $N_{b}$ is less than one or when both are less than one. For the purpose of illustration, a rapid decrease in the temperature is observed in Figures 6-7 when both $N_{t}$ and $N_{b}$ are in the range of 0.1-0.9, while distinct behaviour is observed for the temperature in Figures 4-5 when either $N_{t}$ or $N_{b}$ exceeds one. However, the nanoparticle concentration profile remains the same as shown in Figures 8-9. 


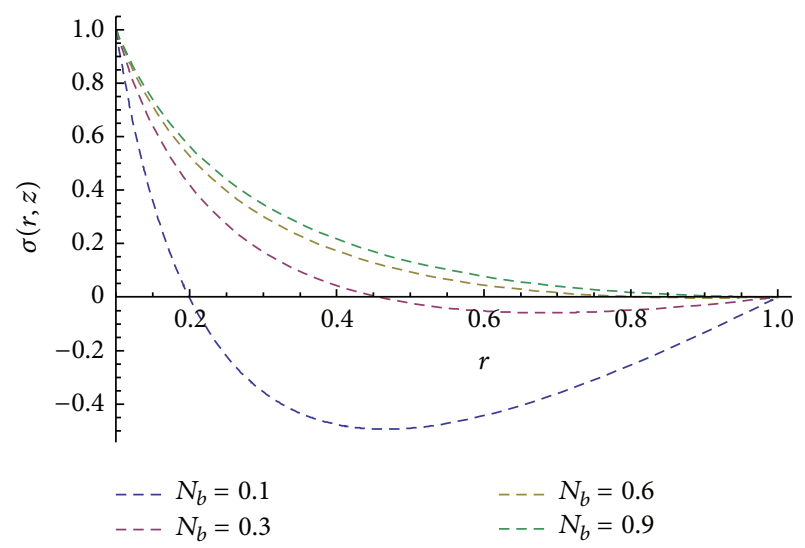

FIGURE 9: Concentration profile for different values of $N_{b}$ at $N_{t}=$ $0.8, r_{1}=0.1, \phi=0.2$, and $z=0.5$.

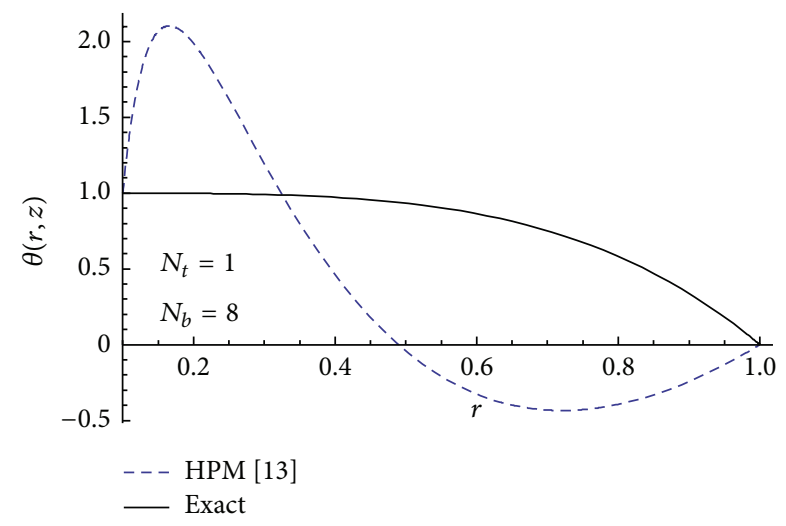

FIGURE 10: Comparison between the HPM and the exact solution for $\theta$ at $N_{t}=1, N_{b}=8, r_{1}=0.1, \phi=0.2$, and $z=0.5$.

Figures 10,11, 12, and 13 display comparisons between the exact temperature solution given by (18) and the approximate temperature solution obtained in [13] by (36). These figures show that the deviation of the approximate solution (HPM) from the exact one for the temperature decreases with decreasing the values of both $N_{t}$ and $N_{b}$. This conclusion is also confirmed through Figures 14, 15, 16, and 17 for the nanoparticle concentration.

As for the rest of the physical quantities such as the pressure gradient and the pressure rise, one can use their exact expressions to obtain several plots which facilitates the physical discussion. Figures 18 to 20 are prepared to see the effect of various parameters on the behavior of the pressure gradient. It is observed from Figure 18 that for $z \in$ $[0,0.45]$ and $z \in[1,1.5]$ the pressure gradient decreases with increasing the amplitude ratio $\phi$, while $d p / d z$ increases with increasing $\phi$ in the other part of the physical domain. Figure 19(a) shows that the pressure gradient is not affected by the variation of the thermophoresis parameter in the range $N_{t} \in[0.1,1.3]$. At higher range for $N_{t} \in[1.5,4.5]$, it is observed from Figure 19(b) that the pressure gradient decreases with increasing $N_{t}$. Both figures show that the maximum value in pressure gradient occurs at $z \approx 0.7$.

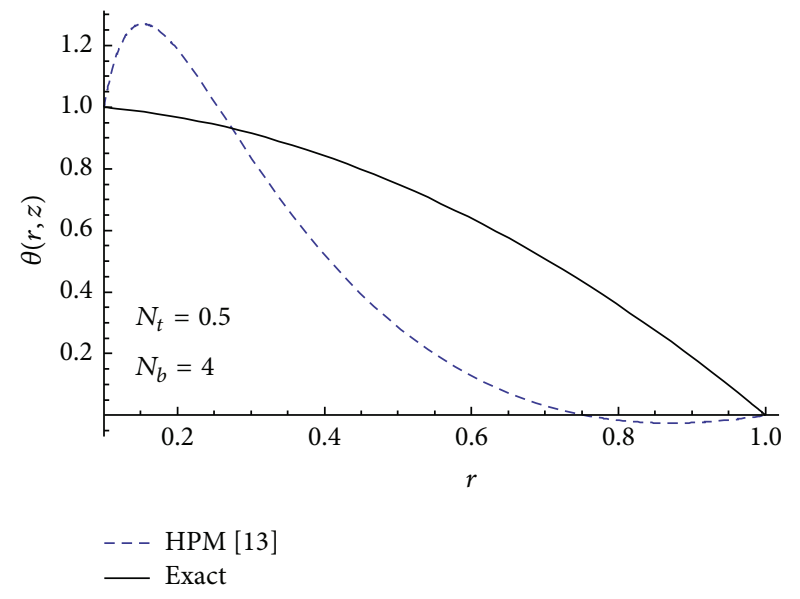

FIGURE 11: Comparison between the HPM and the exact solution for $\theta$ at $N_{t}=0.5, N_{b}=4, r_{1}=0.1, \phi=0.2$, and $z=0.5$.

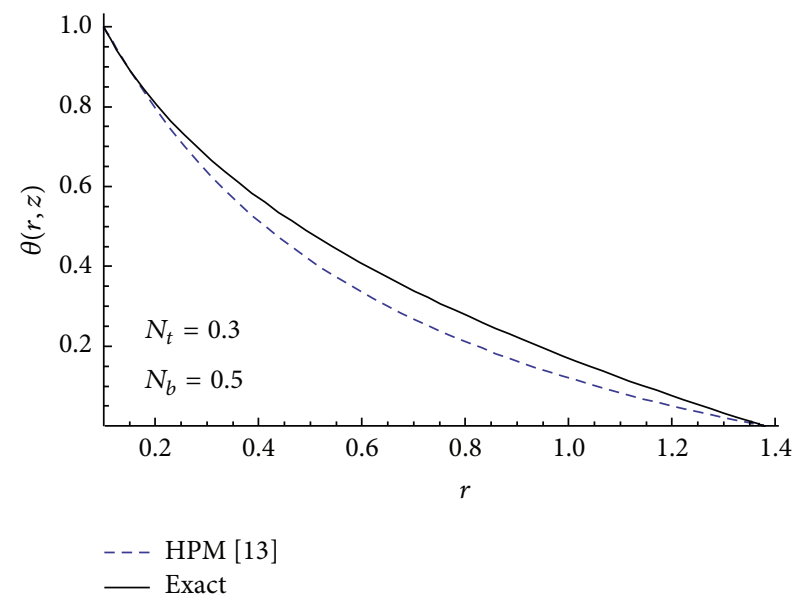

FIGURE 12: Comparison between the HPM and the exact solution for $\theta$ at $N_{t}=0.3, N_{b}=0.5, r_{1}=0.1, \phi=0.4$, and $z=0.3$.

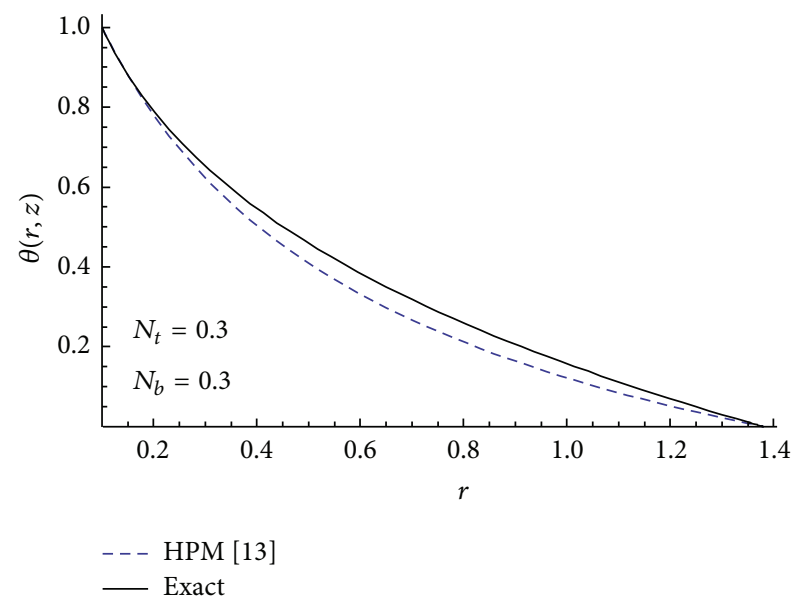

FIGURE 13: Comparison between the HPM and the exact solution for $\theta$ at $N_{t}=0.3, N_{b}=0.3, r_{1}=0.1, \phi=0.4$, and $z=0.3$. 


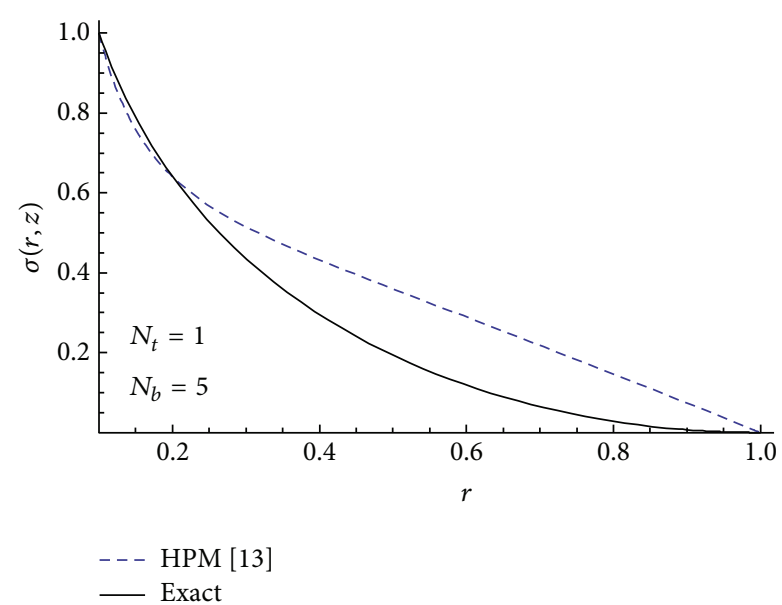

FIGURE 14: Comparison between the HPM and the exact solution for $\sigma$ at $N_{t}=1, N_{b}=5, r_{1}=0.1, \phi=0.2$, and $z=0.5$.

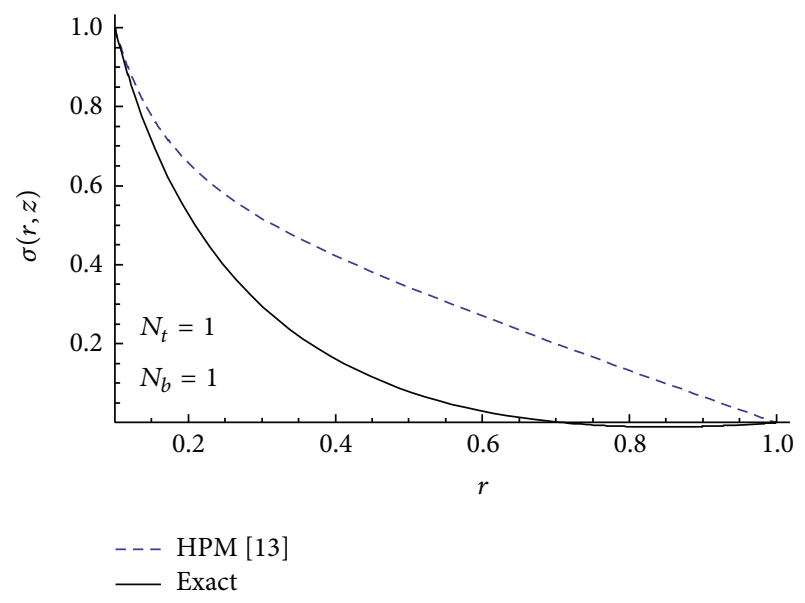

FIGURE 15: Comparison between the HPM and the exact solution for $\sigma$ at $N_{t}=1, N_{b}=1, r_{1}=0.1, \phi=0.2$, and $z=0.5$.

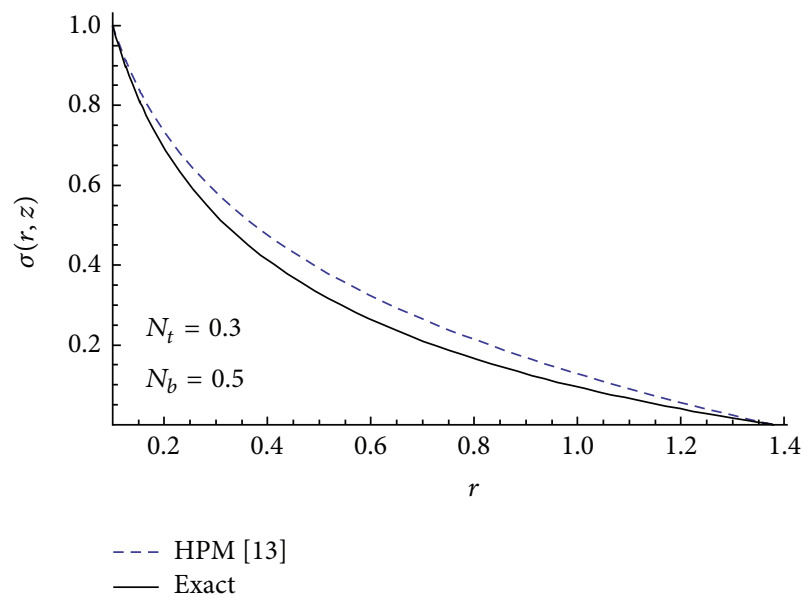

FIGURE 16: Comparison between the HPM and the exact solution for $\sigma$ at $N_{t}=0.3, N_{b}=0.5, r_{1}=0.1, \phi=0.4$, and $z=0.3$.

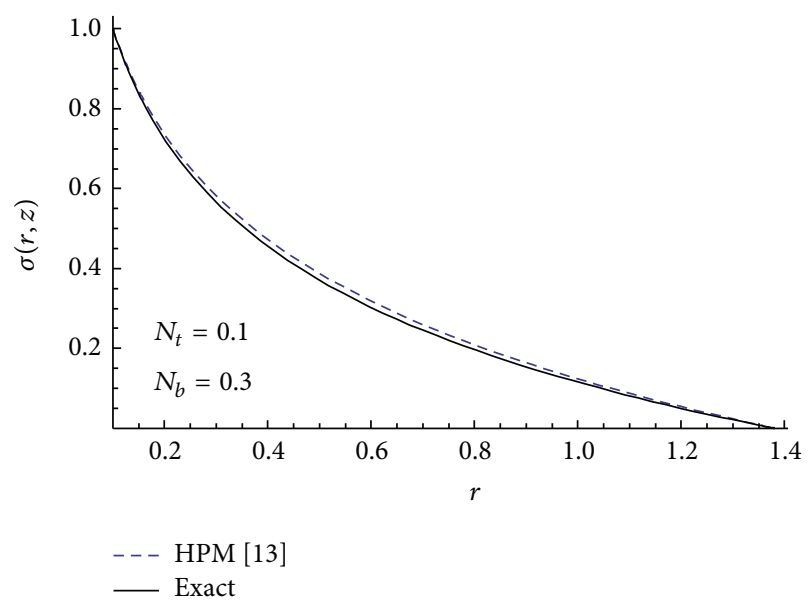

FIGURE 17: Comparison between the HPM and the exact solution for $\sigma$ at $N_{t}=0.1, N_{b}=0.3, r_{1}=0.1, \phi=0.4$, and $z=0.3$.

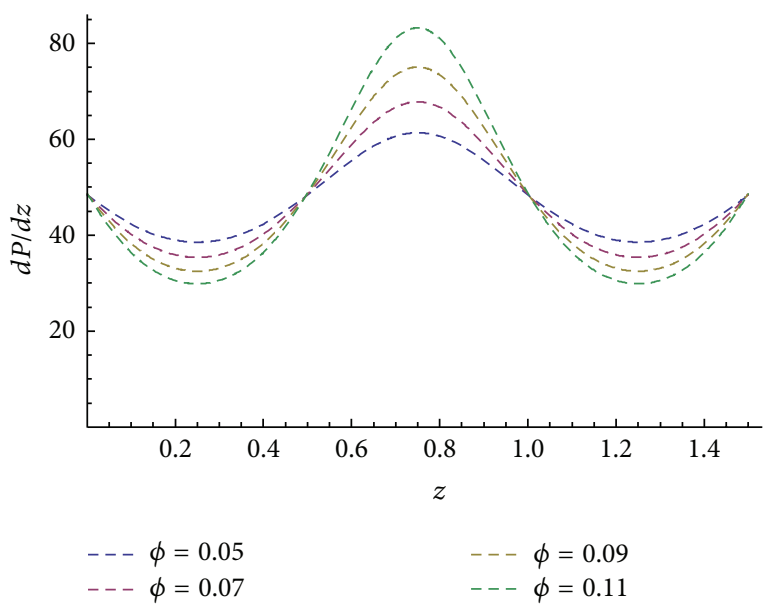

FIGURE 18: Effect of $\phi$ on pressure gradient for $r_{1}=0.05 ; Q=-2$; $N_{t}=0.3 ; G_{r}=0.3 ; B_{r}=0.2 ; N_{b}=0.3$.

The effect of the internal radius $r_{1}$ of the endoscope on the pressure gradient is depicted in Figure 20 which indicates that the pressure gradient increases as $r_{1}$ increases.

The effects of various parameters on the pressure rise $\Delta P$ against volume flow rate $Q$ are shown in Figures 21 to 23 for various values of the amplitude ratio $\phi$, the thermophoresis parameter $N_{t}$, and the radius ratio $r_{1}$. It is observed from Figures 21 to 23 that the pressure rise increases with the increase in the amplitude ratio $\phi$ and the radius ratio $r_{1}$, while the pressure rise decreases with the increase in the thermophoresis parameter $N_{t}$ as shown in Figure 22(b). Unfortunately, Figure 22(a) shows that the pressure rise is not affected by the small variation of $N_{t}$ and this behaviour is different than the one obtained in Figure 2(a) [13]. Finally, it is observed that the solutions derived from the homotopy perturbation method in [13] can be considered accurate when $N_{t}$ and $N_{b}$ take values in short range, less than 0.5 , as described in Figure 17. In order to increase the accuracy of the solution derived from the homotopy perturbation method, it is necessary to increase the number of terms in 


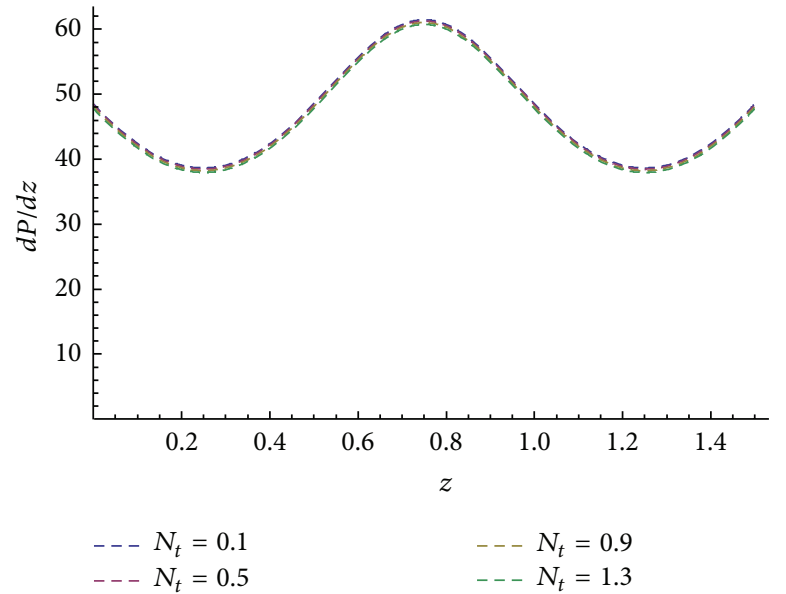

(a)

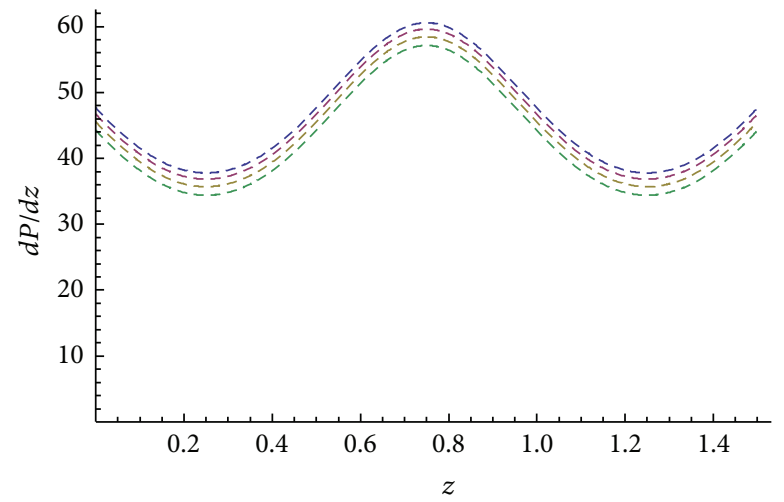

$--N_{t}=1.5$

$---N_{t}=2.5$

$--N_{t}=3.5$

$-N_{t}=4.5$

(b)

FIGURE 19: Effect of $N_{t}$ on pressure gradient for $r_{1}=0.05 ; \phi=0.05 ; Q=-2 ; G_{r}=0.3 ; B_{r}=0.2 ; N_{b}=0.3$.

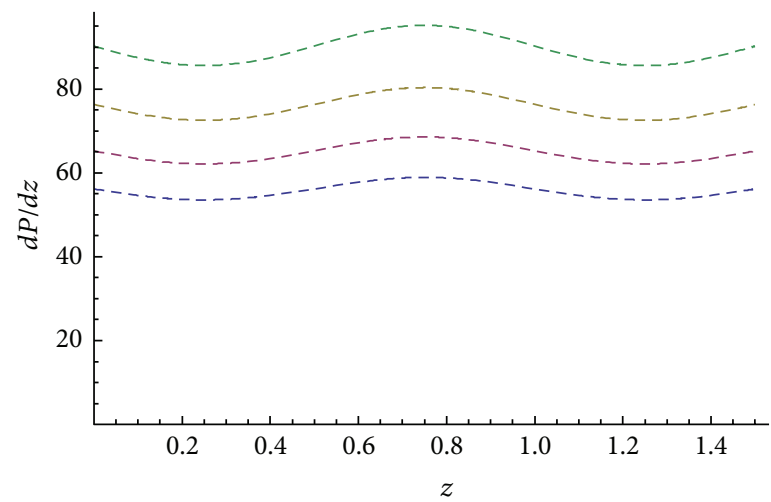

$$
\begin{array}{ll}
--r_{1}=0.10 & --r_{1}=0.20 \\
--- & r_{1}=0.15
\end{array}
$$

FIGURE 20: Effect of $r_{1}$ on pressure gradient for $\phi=0.01 ; Q=-2 ; G_{r}=0.3 ; B_{r}=0.2 ; N_{b}=0.3 ; N_{t}=0.3$.

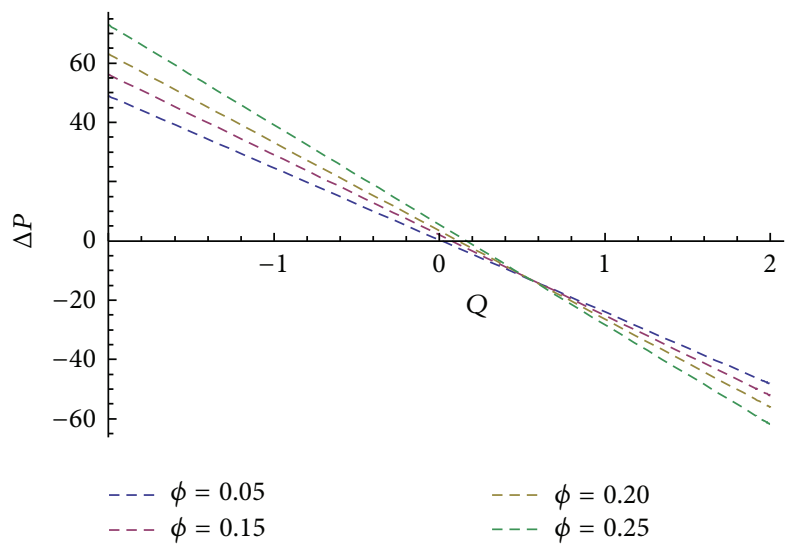

FIgURE 21: Effect of $\phi$ on pressure rise for $r_{1}=0.05 ; G_{r}=0.3 ; B_{r}=0.2 ; N_{t}=0.8 ; N_{b}=0.3$. 


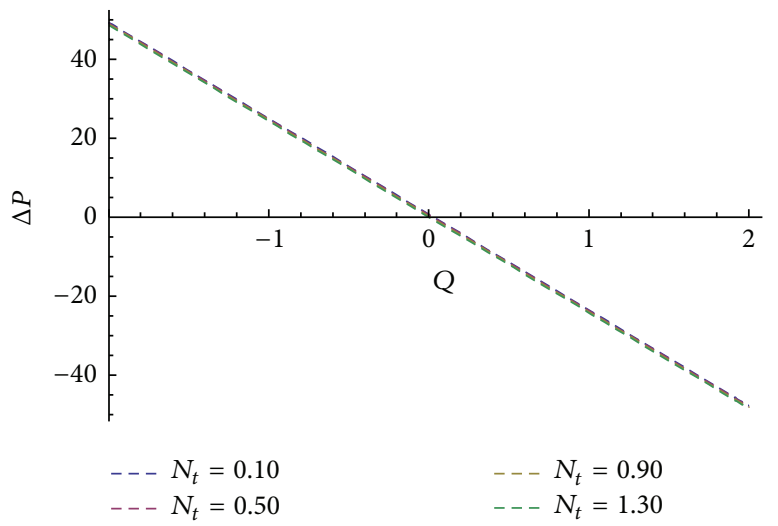

(a)

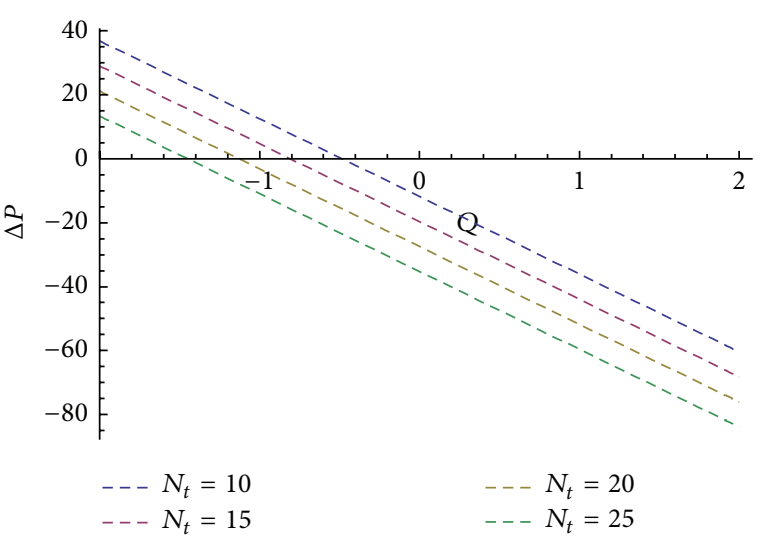

(b)

FiguRE 22: Effect of $N_{t}$ on pressure rise for $r_{1}=0.05 ; \phi=0.05 ; G_{r}=0.3 ; B_{r}=0.2 ; N_{b}=0.3$.

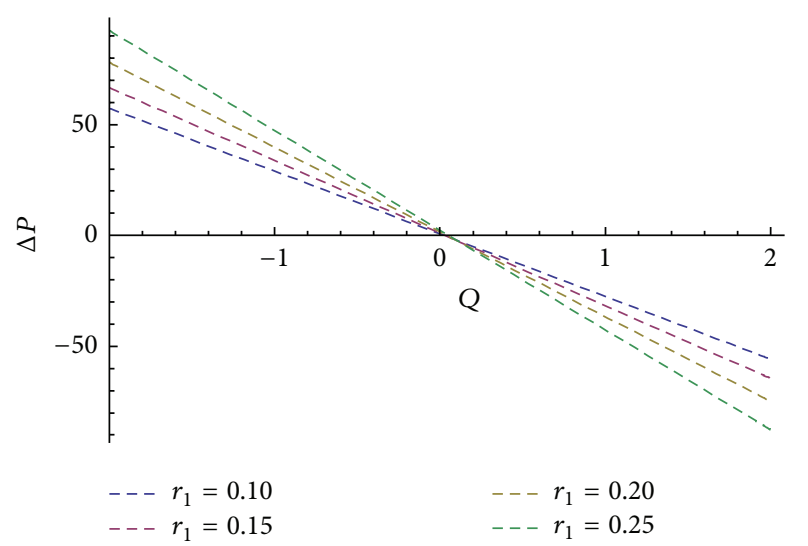

FIGURE 23: Effect of $r_{1}$ on pressure rise for $\phi=0.05 ; G_{r}=0.3 ; B_{r}=$ $0.2 ; N_{t}=15 ; N_{b}=10$.

the series solution. So, it is important to take this conclusion into account in any future work when using homotopy perturbation method. About the number of terms required to obtain accurate numerical solution, several approximate solutions should be obtained till the same curve occurs.

\section{Conclusion}

In this paper, the system of partial differential equations describing the peristaltic flow of a nanofluid with an endoscope has been solved exactly. The obtained exact solutions have been used to study the effects of the thermophoresis parameter $N_{t}$, the Brownian motion parameter $N_{b}$, and other parameters on the temperature, the nanoparticle concentration profiles, the pressure gradient, and the pressure rise. On comparing our exact results with those obtained in [13], remarkable difference in the behaviour of the physical quantities is detected. This may refer to the fact that the approximate solutions obtained in [13] were not sufficient to obtain the correct physical interpretations of the involved phenomena. A final note on the current comparative study is that when it is difficult to get the exact solutions of the physical problem, we instead search for the approximate solutions by using any of the series methods [31, 32], which must be used very carefully in this case in order to achieve the desirable accuracy.

\section{Conflict of Interests}

The authors declare that there is no conflict of interests regarding the publication of this paper.

\section{Acknowledgment}

The authors would like to acknowledge the financial support for this work from the Deanship of Scientific Research (DSR), University of Tabuk, Tabuk, Saudi Arabia, under Grant no. $\mathrm{S} / 82 / 1435 \mathrm{H}$.

\section{References}

[1] T. W. Latham, Fluid motion in a peristaltic pump [M.S. thesis], MIT, Cambridge, Mass, USA, 1966.

[2] S. E. Charm and G. S. Kurland, Blood Flow and MicroCirculation, John Wiley \& Sons, New York, NY, USA, 1974.

[3] T. D. Brown and T.-K. Hung, "Computational and experimental investigations of two-dimensional nonlinear peristaltic flows," Journal of Fluid Mechanics, vol. 83, no. 2, pp. 249-272, 1977.

[4] L. M. Srivastava, V. P. Srivastava, and S. N. Sinha, "Peristaltic transport of a physiological fluid. Part I. Flow in non-uniform geometry," Biorheology, vol. 20, no. 2, pp. 153-166, 1983.

[5] L. M. Srivastava and V. P. Srivastava, "Peristaltic transport of a power-law fluid: application to the ductus efferentes of the reproductive tract," Rheologica Acta, vol. 27, no. 4, pp. 428-433, 1988.

[6] A. Ebaid, "A new numerical solution for the MHD peristaltic flow of a bio-fluid with variable viscosity in a circular cylindrical tube via Adomian decomposition method," Physics Letters A, vol. 372, no. 32, pp. 5321-5328, 2008. 
[7] A. Ebaid and S. M. Khaled, "An exact solution for a boundary value problem with application in fluid mechanics and comparison with the regular perturbation solution," Abstract and Applied Analysis, vol. 2014, Article ID 172590, 7 pages, 2014.

[8] K. S. Mekheimer and Y. Abd elmaboud, "The influence of heat transfer and magnetic field on peristaltic transport of a Newtonian fluid in a vertical annulus: application of an endoscope," Physics Letters, Section A: General, Atomic and Solid State Physics, vol. 372, no. 10, pp. 1657-1665, 2008.

[9] S. B. Field and C. Franconi, Physics and Technology of Hyperthermia, Martinus Nijhoff Publishers, Dordrecht, The Netherlands, 1987.

[10] S. U. S. Choi, "Enhancing thermal conductivity of fluids with nanoparticles," in Proceedings of the ASME International Mechanical Engineering Congress and Exposition, ASME, FED 231/MD, 66, p. 99, San Francisco, Calif, USA, 1995.

[11] S. U. S. Choi, Z. G. Zhang, W. Yu, F. E. Lockwood, and E. A. Grulke, "Anomalous thermal conductivity enhancement in nanotube suspensions," Applied Physics Letters, vol. 79, no. 14, pp. 2252-2254, 2001.

[12] J. Buongiorno and W. Hu, "Nanofluid coolants for advanced nuclear power plants," in Proceedings of the International Congress on Advances in Nuclear Power Plants (ICAPP '05), Paper no. 5705, Seoul, Republic of Korea, May 2005.

[13] N. S. Akbar and S. Nadeem, "Endoscopic effects on peristaltic flow of a nanofluid," Communications in Theoretical Physics, vol. 56, no. 4, pp. 761-768, 2011.

[14] N. S. Akbar, S. Nadeem, T. Hayat, and A. A. Hendi, "Peristaltic flow of a nanofluid in a non-uniform tube," Heat and Mass Transfer, vol. 48, no. 3, pp. 451-459, 2012.

[15] N. S. Akbar and S. Nadeem, "Peristaltic flow of a Phan-ThienTanner nanofluid in a diverging tube," Heat Transfer-Asian Research, vol. 41, no. 1, pp. 10-22, 2012.

[16] M. Mustafa, S. Hina, T. Hayat, and A. Alsaedi, "Influence of wall properties on the peristaltic flow of a nanofluid: analytic and numerical solutions," International Journal of Heat and Mass Transfer, vol. 55, no. 17-18, pp. 4871-4877, 2012.

[17] N. S. Akbar, S. Nadeem, T. Hayat, and A. A. Hendi, "Peristaltic flow of a nanofluid with slip effects," Meccanica, vol. 47, no. 5, pp. 1283-1294, 2012.

[18] A. Ebaid and E. H. Aly, "Exact analytical solution of the peristaltic nanofluids flow in an asymmetric channel with flexible walls and slip condition: application to the cancer treatment," Computational and Mathematical Methods in Medicine, vol. 2013, Article ID 825376, 8 pages, 2013.

[19] E. H. Aly and A. Ebaid, "Effect of the velocity second slip boundary condition on the peristaltic flow of nanofluids in an asymmetric channel: exact solution," Abstract and Applied Analysis, vol. 2014, Article ID 191876, 11 pages, 2014.

[20] E. H. Aly and A. Ebaid, "Exact analytical solution for the peris taltic flow of nanofluids in an asymmetric channel with slip effect of the velocity, temperature and concentration," Journal of Mechanics, vol. 30, no. 4, pp. 411-422, 2014.

[21] N. S. Akbar, "MHD Eyring-Prandtl fluid flow with convective boundary conditions in small intestines," International Journal of Biomathematics, vol. 6, no. 5, Article ID 1350034, 1350034, 13 pages, 2013.

[22] N. S. Akbar and S. Nadeem, "An analytical and numerical study of peristaltic transport of a Johnson-segalman fluid in an endoscope," Chinese Physics B, vol. 22, no. 1, Article ID 014703, 2013.
[23] N. S. Akbar, S. Nadeem, and C. Lee, "Characteristics of Jeffrey fluid model for peristaltic flow of chyme in small intestine with magnetic field," Results in Physics, vol. 3, pp. 152-160, 2013.

[24] N. S. Akbara and S. Nadeemb, "Mixed convective magnetohydrodynamic peristaltic flow of a jeffrey nanofluid with newtonian heating," Zeitschrift fur Naturforschung A, vol. 68, no. 6-7, pp. 433-441, 2013.

[25] N. S. Akbar and S. Nadeem, "Nano sutterby fluid model for the peristaltic flow in small intestines," Journal of Computational and Theoretical Nanoscience, vol. 10, pp. 2491-2499, 2013.

[26] S. Nadeem, S. Ijaz, and N. S. Akbar, "Nano particle analysis for the steady blood flow of jeffrey fluid with stenosis with new analytical techniques," Journal of Computational and Theoretical Nanoscience, vol. 10, no. 11, pp. 2751-2765, 2013.

[27] N. Sher Akbar and S. Nadeem, "Biomathematical study of nonNewtonian nanofluid in a diverging tube," Heat Transfer-Asian Research, vol. 42, no. 5, pp. 389-402, 2013.

[28] P. Rana and R. Bhargava, "Flow and heat transfer of a nanofluid over a nonlinearly stretching sheet: a numerical study," Communications in Nonlinear Science and Numerical Simulation, vol. 17, no. 1, pp. 212-226, 2012.

[29] O. D. Makinde and A. Aziz, "Boundary layer flow of a nanofluid past a stretching sheet with a convective boundary condition," International Journal of Thermal Sciences, vol. 50, no. 7, pp. 13261332, 2011.

[30] E. H. Aly, A. Ebaid, and N. Y. Abd Elazem, "Analytical and numerical investigations for the flow and heat transfer of nanofluids over a stretching sheet with partial slip boundary condition," Applied Mathematics \& Information Sciences, vol. 8, no. 4, pp. 1639-1645, 2014.

[31] N. T. Eldabe, E. M. Elghazy, and A. Ebaid, "Closed form solution to a second order boundary value problem and its application in fluid mechanics," Physics Letters A, vol. 363, no. 4, pp. 257-259, 2007.

[32] A. Ebaid, "Approximate analytical solution of a nonlinear boundary value problem and its application in fluid mechanics," Zeitschrift fur Naturforschung-Section A Journal of Physical Sciences, vol. 66, no. 6-7, pp. 423-426, 2011. 


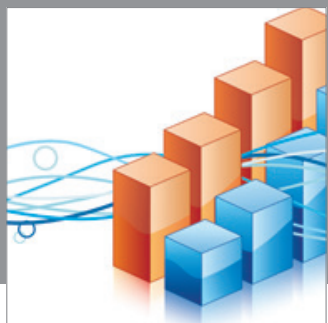

Advances in

Operations Research

mansans

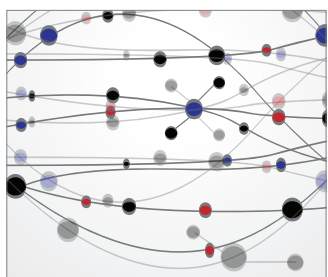

The Scientific World Journal
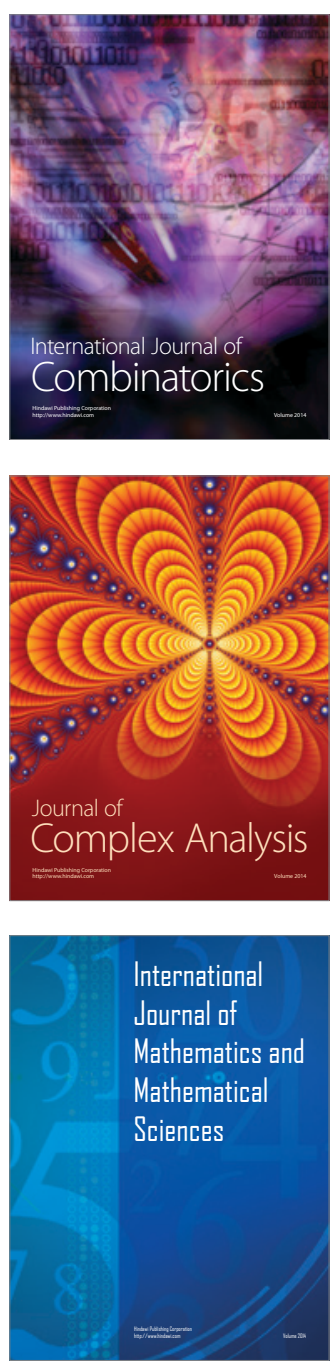
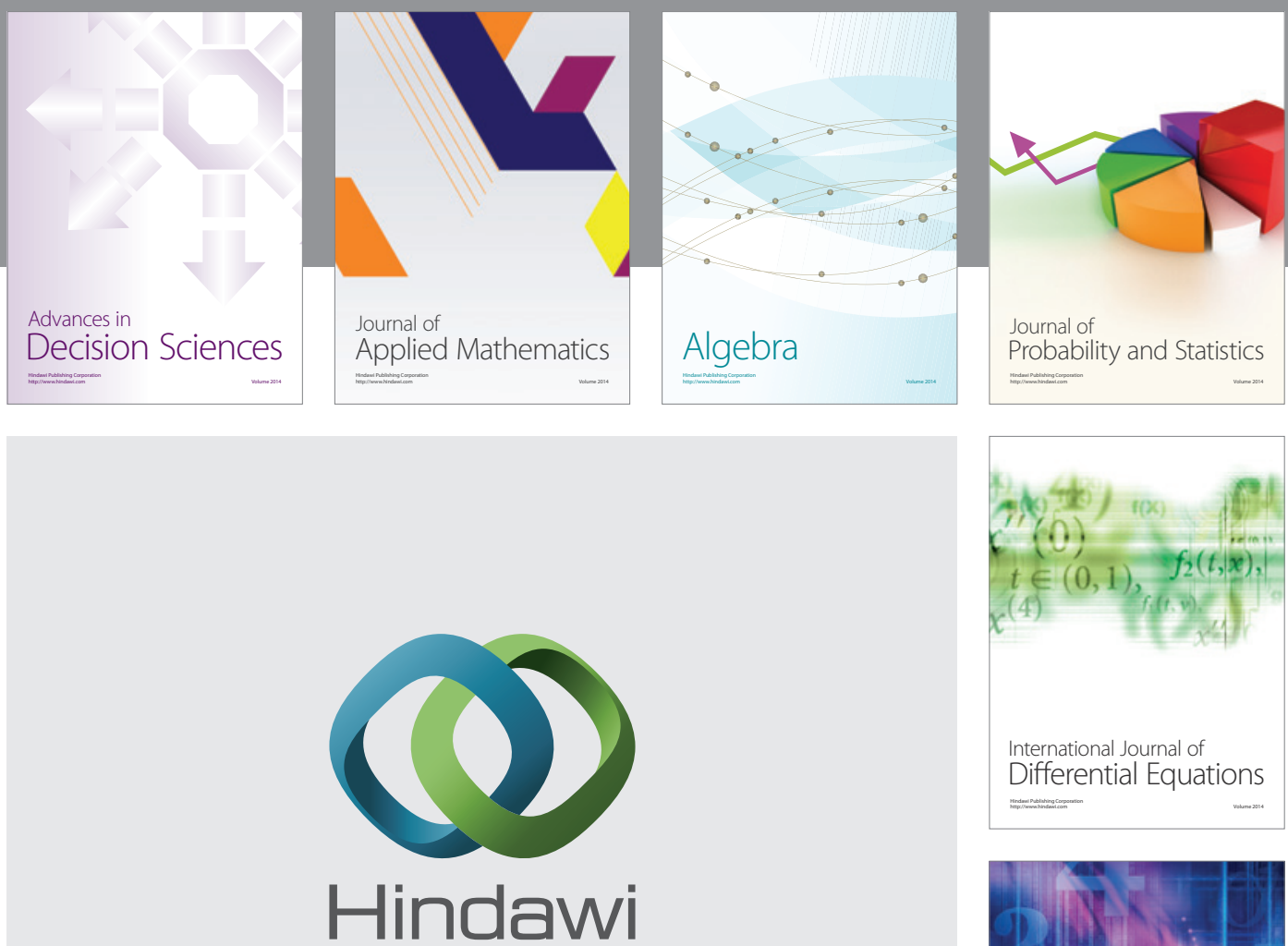

Submit your manuscripts at http://www.hindawi.com
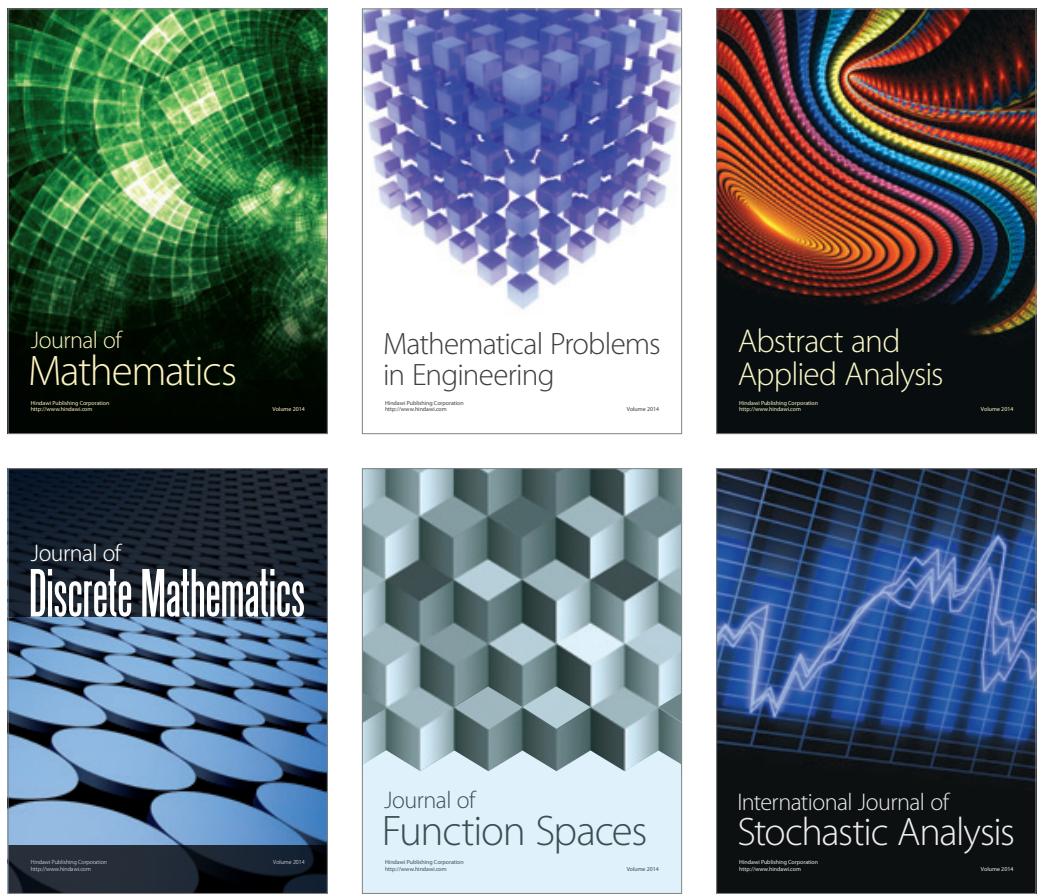

Journal of

Function Spaces

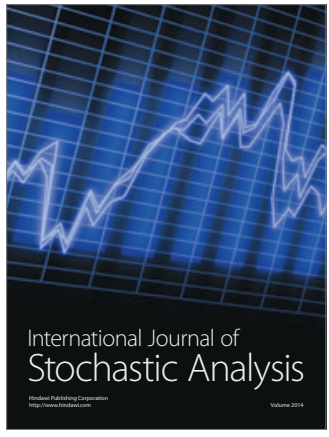

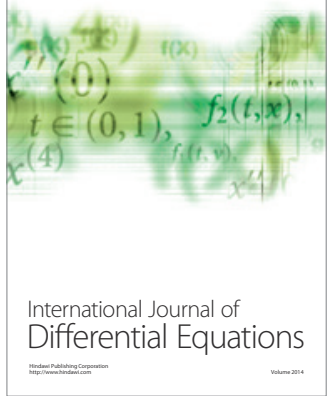
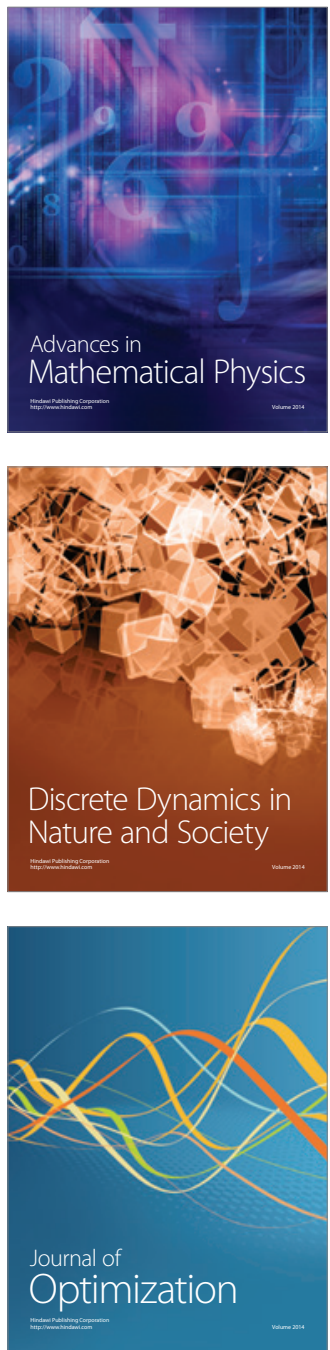